\title{
Medienerziehung in der Erwachsenenbildung
}

von Georg Betz

Erwachsenenbildung in katholischer Trägerschaft, die in der Bundesrepublik Deutschland über ein nahezu flächendeckendes Organisationsnetz verfügt, hat Medienerziehung verbal zu einem ihrer Arbeitsschwerpunkte erklärt. Schon die grobe Analyse der Leistungsstatistiken und Veranstaltungsverzeichnisse kann jedoch zeigen, daß - bei aller Differenziertheit der Programmsituation Anspruch und Wirklichkeit zumindest vor Ort, außerhalb der Bildungshäuser, sehr weit auseinanderklaffen ${ }^{1}$ :

- Medienpädagogische Maßnahmen stellen im örtlichen Bildungsangebot eine große Rarität dar. Ihr Anteil am Gesamt aller Aktivitäten katholischer Erwachsenenbildung ist verschwindend gering.

- Beim ohnehin bescheidenen Engagement handelt es sich zumeist um Einzelabende. Lernintensive mehrteilige Bildungseinheiten sind der örtlichen Programmpraxis so gut wie gänzlich fremd.

- Das medienpädagogische Veranstaltungsprogramm wird weder systematisch und kontinuierlich geplant noch regional gezielt gestreut. Es wirkt zufällig und okkasionell.

- Mit Abstand die meiste Aufmerksamkeit findet bei den Programmachern das Fernsehen. Presse, Hörfunk oder der Film sind nur selten Gegenstand von Veranstaltungen.

- Medien werden vorwiegend als potentielle Störfaktoren der kindlichen Sozialisation gesehen. Als Vermittler des Zeitgesprächs der Gesellschaft treten sie vor Ort in den Bildungsprogrammen nur selten in Erscheinung.

- Aktuelle Vorgänge in der Medienlandschaft und -politik werden so gut wie nicht oder allenfalls mit großer zeitlicher Verzögerung in den Bildungsprogrammen vor Ort aufgegriffen.

Bekanntlich erweist sich das Gefüge von Einstellungen und Gewohnheiten gegenüber dem pädagogischen Bemühen, gegenüber kommunikativen Veränderungsversuchen als sehr stabil ${ }^{2}$. Der bescheidene Umfang der medienpädagogischen Aktivitäten vor Ort, ihre Diskontinuität, die Verengung ihrer Zielperspektive, ihre geringe Intensität, die wiederum lernbeeinträchtigende methodische $Z$ wänge schafft - all das drängt massive Zweifel an einem nennenswerten nachhaltigen Ertrag des medienpädagogischen Engagements katholicher Erwachsenenbildung auf.

Kurzum, in Quantität und Qualität bleibt die Medienerziehung in der katholischen Erwachsenenbildung weit hinter den von der Pastoralinstruktion „Communio et progressio" entwickelten Grundsätzen zurück. Und noch bedenklicher: In der Längsschnittanalyse der Leistungsstatistiken wie der Programmverzeichnisse sind keine Anzeichen für eine breitenwirksame Änderung dieses Zustands auszu-

Dr. Georg Betz ist wissenschaftlicher Assistent am Lehrstuhl Erwachsenenbildung der Katholischen Universität Eichstätt und Vorsitzender eines katholischen Kreisbildungswerkes. 
machen. Medienpädagogik vegetiert seit Jahren ganz am Rand des Bildungsangebots katholischer Erwachsenenbildung in den Gemeinden und Landkreisen in didaktisch fragwürdiger Weise vor sich hin.

Dem steht ein greifbarer Ausbau der Infrastrukturen zur Forcierung und Qualifizierung der kirchlichen Aktivitäten im Feld der Medienarbeit gegenüber: Da hat es personelle Erweiterung in den Medienzentralen auf Diözesanebene gegeben. Oder da wäre insbesondere auf die Errichtung des Referats Kommunikationspädagogik bei der Zentralstelle Medien der deutschen Bischofskonferenz zu verweisen, das via breitgestreuter verschriftlichter Veranstaltungsanregungen, per Fortbildungsmaterial sowie intensiver Angebote zur Schulung von Referenten medienpädagogische Breitenarbeit anzukurbeln sucht. Die Verstärkung zentraler Förderinstanzen scheint freilich im örtlichen Bildungsangebot bislang noch keinen erkennbaren Niederschlag gefunden zu haben.

Ebensowenig scheinen die organisatorische Neuordnung und die Professionalisierung katholischer Erwachsenenbildung in den 70er Jahren die Medienerziehung vor Ort nennenswert tangiert zu haben. Das in Reaktion auf die einschneidenden bildungspolitischen wie theoretischen Umbrüche in der Weiterbildungslandschaft wie in Reaktion auf den vom Konzil erneuerten Weltdienstauftrag der Kirche entwickelte Ausbaukonzept katholischer Erwachsenenbildung wollte mit der Errichtung von Bildungswerken und der Anstellung ihnen zugeordneter hauptamtlicher Mitarbeiter nicht zuletzt die Aktivitäten vor Ort verstärken und qualifizieren. Die strukturelle Neuordnung ist sehr weit fortgeschritten, die Mitarbeiterzahlen haben sich in den 70er Jahren vervielfacht, die Zuschüsse auch. Die Medienerziehung freilich stagniert, ja sie ist gemessen an der Expansion der sonstigen Aktivitäten eher noch weiter an den Rand gerückt.

Allerdings - und das weist der Suche nach Ursachen schon wichtigere Spuren - finden sich die das medienpädagogische Angebot kennzeichnenden bedenklichen Schwächen und Fragwürdigkeiten wie ihre Stabilität zum Teil auch in anderen Teilfeldern katholischer Erwachsenenbildung. Zufälligkeit, Punktualität, Kurzatmigkeit herrschen auch in der Elternbildung, der theologischen Erwachsenenbildung oder der politischen Bildung vor. Letzere nimmt überdies gemessen an der zentralen Bedeutung, die sie in offiziellen Zielaussagen innehat, im faktichen örtlichen Bildungsangebot ebenfalls nur eine Randstellung ein ${ }^{3}$. Insofern wird man nach Bedingungen der Desolatheit des medienpädagogischen Angebots in der katholischen Erwachsenenbildung vor allem auch außerhalb des spezifischen Umfelds der Medienthematik suchen müssen.

Das medienpädagogische Veranstaltungsangebot ist wie das Zustandekommen des Gesamtprogramms Resultat, Produkt eines sehr komplexen Vorgangs. Viele Einflüsse, Überlegungen, Instanzen bestimmen ihn direkt oder indirekt mehr oder minder stark mit. Dieses komplexe Variablengeflecht läßt sich derzeit nicht voll entwirren und auch nicht auf die Größenordnung einzelner Einflußfaktoren hin abtaxieren. Die empirische Durchdringung der Realität katholischer Erwachsenenbildung vor Ort steckt noch in den allerersten Anfängen. Deshalb kann auch dem folgenden Versuch, den Hintergrund der Riesenkluft zwischen Anspruch und Wirklichkeit der Medienpädagogik in der katholischen Erwachsenenbildung zu erhellen, nur hypothetischer Charakter zukommen, die Funktion des pointierten Impulses zur weiteren differenzierenden Diskussion und Bearbeitung. 


\section{Fragreürdigkeiten in der Verortung der Programmentscheidung}

Die Suche nach Bedingungen des defizitären Zustands der Medienpädagogik im örtlichen Programm katholischer Erwachsenenbildung wird zweckmäßigerweise dort ansetzen, wo eben dieses Programm erstellt wird: vor Ort. Im organisatorischen Gefüge katholischer Erwachsenenbildung ist die jeweilige Gemeinde ihr eigener Programmsouverän. Auf dem Papier nimmt dabei der Pfarrgemeinderat die Funktion des ,gatekeepers“ wahr, der darüber befindet, was in welcher Ausführlichkeit wann usw. in der Gemeinde thematisiert wird und was nicht. Vielerorts freilich werden die Vorentscheidungen bereits in einem dem Pfarrgemeinderat zugeordneten Sachausschuß Erwachsenenbildung gefällt. Und häufig genug ist die gesamte Verantwortung für den Aufgabenbereich Erwachsenenbildung auch nur an einen einzigen Beauftragten delegiert. .

Diese dezentralisierte Verortung der didaktischen Entscheidungen in der katholischen Erwachsenenbildung entspricht dem in der Weiterbildungsdiskussion oft vorgebrachten Postulat der Verbrauchernähe der Planung, die orts- und regionalspezifische Bedürfnisse miteinbeziehen soll. Zugleich freilich konstituiert diese Verortung eine hochgradige Abhängigkeit katholischer Bildungsarbeit vom Engagement und vom Sachverstand der (des) örtlichen Mitarbeiter(s). Erste systematische Versuche, diese Abhängigkeit einmal differenzierter zu qualifizieren ${ }^{4}$, stimmen stark skeptisch und lassen bezogen auf das faktische medienpädagogische Angebot dessen stabilen defizitären Zustand zu einem nicht geringen Teil erklären.

Auch wenn die Wirklichkeit vor Ort sehr unterschiedlich aussieht: Erwachsenenbildung hat vor Ort, in den Gemeinden alles in allem nur einen relativ geringen Stellenwert. Vielerorts wird sie als lästiges Übel empfunden. Wer dafür verantwortlich zeichnet, tut das häufig nur deshalb, weil sich kein anderer dazu bereitgefunden hat. Oft genug kann er sich der übernommenen Aufgabe auch nicht mit dem nötigen Engagement widmen, weil er mit Doppel- und Dreifachfunktionen belastet ist. In aller Regel haben die örtlichen Mitarbeiter keine spezifische Vorbereitung für ihre Tätigkeit. Viele scheinen das auch gar nicht als Defizit zu empfinden. Die zeitliche Belastung bedrückt mehr als die inhaltlich-didaktische Anforderung. Es herrscht vor Ort weithin noch tiefe Bewußtlosigkeit der Aufgabe gegenüber.

Kommt hinzu, daß häufig das Aufgabenverständnis der Programmacher vor Ort mit den erklärten Zielsetzungen an der Spitze katholischer Erwachsenenbildung konfligiert. Sofern Erwachsenenbildung überhaupt pädagogisch verstanden wird, sofern über Veranstaltungen überhaupt Lernprozesse initiiert, Einblick vertieft, Bewußtsein geweckt, Fähigkeit mobilisiert werden soll, leitet nicht selten ein eng gehaltenes pastorales bzw. katechetisches Interesse die Programmplanung. Das Veranstaltungsprogramm wird vorrangig zum Kanal des Versuchs der wirksameren Verkündigung christlicher Lehre, Wertvorstellungen und Verhaltensnorm.

Nur zu häufig freilich wird in den Pfarrgemeinden das Veranstaltungsprogramm der Erwachsenenbildung primär auch nur als Instrument zur rein äußeren Verlebendigung des Pfarrlebens genutzt. Erwachsenenbildung wird in den Dienst der Bereicherung des außergottesdienstlichen Geselligkeits- und Kontaktangebots der Pfarrei gestellt. Ihrer pädagogischen Funktion geht sie dabei verlustig. Hilfe bei der Bewältigung der Zumutungen und Anforderungen in den verschiedenen Lebensbereichen, wie sie das Weltdienst-Verständnis der katholischen Erwachsenenbildung impliziert, wird mit den Veranstaltungen nicht bezweckt. 
Medienerziehung hat es bei diesem Bewußtseinsstand schwer, in das Blickfeld der Programmacher vor Ort zu treten, zumal diese weithin noch stark in volkskirchlichen Kategorien denken, in denen die Quantität der Nutzer des kirchlichen Veranstaltungshandelns eine bedeutsamere Rolle spielt als die Qualität der Nutzung. Entsprechend sieht der Leistungs- und Erfolgsbegriff an der Basis aus. Er bestimmt sich weithin von der Teilnehmerzahl her. Ihrer Antizipation wird folglich bei der didaktischen Planung besonderer Vorrang gegeben.

Die Neigung, publikumsattraktiv zu planen, damit viel Gemeinde, Kirche zusammenkommt, wird dadurch noch erheblich verstärkt, daß bislang nur eine Minderheit die Notwendigkeit erkennt und bereit ist, sich weiterzubilden ${ }^{5}$. Und diese Minderheit schmilzt nochmals stark zusammen, wenn die Bereitschaft gefragt ist, Fortbildungsangebote zu nutzen, die erstens nicht beruflich verwertbar sind und zweitens von der Kirche und womöglich noch in kirchlichen Räumen offeriert werden.

Die programmatisch als Lebens- und Orientierungshilfe angetretene Weiterbildung gerät damit vor Ort stark in den Sog des Marktes. Was häufig nachgefragt wird, hat auch die besten Chancen, ins Programm zu kommen. Die Frage nach der Bildungsrelevanz tritt demgegenüber in den Hintergrund. Somit bleibt vieles, was von der Lebenssituation der Teilnehmer her objektiv stärker der Thematisierung bedürfte, was existentiell bedeutsamer wäre, auf der Strecke. Neue Ziele, neue Qualifikationen sind gegenüber dem Gängigen nur sehr mühsam durchzudrücken.

Unter anderem steht Medienerziehung vor dieser Schwelle. Sie gehört nicht zu den etablierten Fächern der Erwachsenenbildung. Und sie hat - die Leistungsstatistiken lassen das durchweg ablesen - (noch) nicht den Stoff, für den sich zur Zeit viele Teilnehmer erwärmen ließen. Je intensiver, längerfristiger medienpädagogische $\mathrm{Maßnahmen}$ angelegt sind, desto mehr schrumpft das potentielle Nutzerreservoir. In kleinen Gemeinden, auf dem Land sind dafür nur sehr wenige Interessenten auf die Beine zu bringen. Diese Aussichten schrecken ganz ab oder lassen allenfalls den Versuch mit einem Einzelabend angeraten sein.

\section{Schwächen im mittleren und böheren Management}

Nur unter konzentriertem Einsatz der verfügbaren Kräfte ließen sich die genannten Barrieren zu mehr und wirksamerer Medienerziehung in den Programmen katholischer Erwachsenenbildung überwinden. Dieser massierte Einsatz müßte angesichts der Bewußtlosigkeit an der Basis zunächst von den mittleren und höheren Ebenen im organisatorischen Gefüge katholischer Erwachsenenbildung kommen. Die freilich dürften dazu derzeit schon vom nötigen Arbeitsaufwand her gesehen nicht in der Lage sein. Denn trotz zahlenmäßig greifbarer Professionalisierung wird die Arbeit vor Ort immer noch im hohen $\mathrm{Ma} B$ von ehrenamtlichen Führungskräften inspiriert. In weiten Landstrichen gibt es überhaupt keine hauptamtlichen Mitarbeiter. Und wo es sie gibt, sind sie vielfach so mit technisch-organisatorischen Aufgaben belastet, daß sie nur in bescheidenem Umfang die Funktion der intensiven pädagogischen Betreuung der Basis übernehmen können.

Die Verzahnung zwischen der Bildungspraxis vor Ort und den zu ihrer Qualifizierung geschaffenen Instanzen ist nicht zureichend. Die Arbeit treibt mehr vor sich hin, als daß sie von den Führungskräften in die selbstgesteckten Zielrichtungen vorangetrieben wird. Das ist freilich nicht nur eine Folge des geringen Professionalisie- 
rungsgrades bzw. des Mangels an Zeit. Unter denen, die in der Struktur katholischer Erwachsenenbildung die Arbeit an der Basis unterstützen, qualifizieren und in die programmatisch vorgezeichneten Bahnen leiten sollen, fehlt es vielmehr auch am dazu erforderlichen Sachverstand.

Viele der in führender Funktion auf der Ebene des Landkreises bis hinauf zur Bundesebene Tätigen bringen bewundernswerten Idealismus mit, zumeist auch solide Kompetenzen im einen oder anderen Stoffgebiet. Ihre Fähigkeit, das Feld Erwachsenenbildung theoretisch zu reflektieren, $\mathrm{zu}$ analysieren und konzeptionell weiterzuentwickeln, ihre makrodidaktische Kompetenz ist dagegen häufig unterentwickelt. Und eine entsprechende Fortbildung des mittleren und höheren Managements steckt im katholischen Weiterbildungslager noch in allerersten Anfängen.

Die Anstellung hauptamtlicher Mitarbeiter hat dieses Sachverstandsdefizit katholischer Erwachsenenbildung nicht zufriedenstellend verringert. Man hat sich vielfach nicht jene sozialwissenschaftlichen und didaktischen Qualifikationen eingekauft, die den anfangs der 70er Jahre gehegten Reformvorstellungen vorschwebten ${ }^{6}$. So sind unter den Bewerbern für die wenigen Hauptamtlichen-Positionen in manchen Diözesen bevorzugt Theologen oder Religionspädagogen zum Zug gekommen.

Zum Teil werden die geschaffenen Stellen auch so gering dotiert, daß sie für die auf eine analysierende, konzipierende und beratende Tätigkeit im sozialen Bereich theoretisch fundierter Ausgebildeten - und das sind vom Studienkonzept her eher Hochschul- als Fachhochschulabsolventen - nicht interessant waren und sind. Ein entsprechendes spezifisches Einführungs- und Fortbildungsprogramm für hauptamtliche Mitarbeiter, welches die Versäumnisse der Einstellungspolitik im Nachhinein entschieden korrigieren konnte, fehlt ebenfalls noch.

Alles in allem - die Personalpolitik ist beredter Ausdruck wie Ursache dafür krankt die Erwachsenenbildung in katholischer Trägerschaft, was die flächendeckende Arbeit außerhalb der Bildungshäuser anlangt, an Kopflosigkeit. Die Reflexion hinkt weit hinter dem Aktivismus einher ${ }^{7}$. Es fehlt an einer Einrichtung, welche die vielfältigen Aktivitäten bundesweit inspiriert und koordiniert. Näher besehen wursteln die Diözesen, die Bildungswerke und andere Instanzen in Sachen Erwachsenenbildung weithin beziehungslos vor sich hin ${ }^{8}$. Die Dachorganisationen auf Landes- oder Bundesebene sind nur lose Zusammenschlüsse, eher auf Interessenvertretung nach außen bedacht als auf Qualifizierung der Arbeit nach innen.

Eine pädagogische Arbeitsstelle, wie sie sich die Volkshochschulen und die evangelische Erwachsenenbildung leisten, die als Scharnier zwischen Wissenschaft und Praxis die aktuelle Diskussion verfolgt, mittragen hilft und vor allem auch nach innen, an die Mitglieder der eigenen Organisation weitervermittelt, um anfallende Weichenstellungen wie die laufende Praxis zu fundieren, war zwar 1975 auf einer Jahrestagung der Katholischen Bundesarbeitsgemeinschaft für Erwachsenenbildung einhellig als notwendig erkannt worden. Bis heute ist freilich diese wichtige Einrichtung nicht errichtet, ja es gibt überhaupt keine Anzeichen dafür, daß sie in absehbarer Zeit kommen wird.

Medienerziehung tut sich also nicht nur schwer, als eine wichtige Aufgabe der Lebens- und Orientierungshilfe ins Bewußtsein der Mitarbeiter an der Basis zu dringen. Sie dürfte bisher als solche auch kaum Eingang im Bewußtsein der 
Führungsschicht katholicher Erwachsenenbildung gefunden haben, geschweige denn, daß dort die Schwächen und Fragwürdigkeiten des faktischen medienpädagogischen Angebots voll erkannt wären. Der Glaube an den zwangsläufigen Zusammenhang zwischen Absicht und Resultat pädagogischen Bemühens ist im Management katholischer Erwachsenenbildung noch ziemlich tief verankert. Die Berater der Programmacher vor Ort bedürfen also selbst noch der massiven Beratung und Inspiration, bis die Medienerziehung die Bedeutung und Qualität erlangen kann, die ihr einige wenige Spitzenfunktionäre und die Theoretiker der Erwachsenenbildung sowie der amtskirchliche Auftrag zugewiesen haben.

\section{Die Wettbewerbssituation im Weiterbildungsbereich}

Die sich abzeichnende Entwicklung zu einer Qualifizierung auch der mittleren und höheren pädagogischen Führungskräfte wird vermutlich auch deren medienpädagogisches Problembewußtsein erweitern und vertiefen. Dennoch bleibt eine Menge Skepsis gegenüber der Prognose, da $ß$ sich dementsprechend auch das medienpädagogische Veranstaltungsangebot verbreitern, intensivieren und qualitativ verbessern wird. Die Skepsis rührt vom Blick auf die Zwänge quantitativen Denkens her, dem sich auch die Vorstände der Bildungswerke, die hauptamtlichen pädagogischen Mitarbeiter, die Führungsspitzen auf Diözesan-, Landes- oder Bundesebene nicht ganz entziehen können. Im Gegenteil, ihnen, denen sich viel stärker als den Mitarbeitern vor Ort Fragen der Finanzierung der Bildungsarbeit stellen, sind stark die Hände gebunden, wenn sie das gängige nachfrageorientierte Programmrepertoire auf breiter Front umstellen wollten.

Zum einen geraten sie in Legitimitätsdruck gegenüber den kirchlichen Zuschußgebern, die ebenfalls weithin noch in den oben genannten volkskirchlichen Kategorien mit dem hohen Gewicht der Besucherzahl und in naiven Vorstellungen von den Wirkmöglichkeiten kommunikativer Beeinflussungsversuche denken, und die zum Teil auch noch nicht den Wert und die Notwendigkeit von nicht unmittelbar kirchen- und glaubensbezogener Weiterbildung in und durch die Kirche voll erkannt haben. In dieser Situation wird die Existenz- und Zuschußberechtigung nach innen eben am ehesten noch in Reichweitedaten und Steigerungsquoten nachgewiesen - umso überzeugender, je höher die Zahlen sind.

Zum anderen geraten die Führungskräfte auf regionaler und vermutlich mehr noch die auf Diözesan- und Landesebene in $Z$ wänge von außen, seitens der kommunalen und staatlichen Förderung und seitens der Bildungspolitik. Gerade die Fessseln, die der Führungsschicht - und zwar aller Weiterbildungsträger - von dieser Seite angelegt werden, engen den Innovationsspielraum in der Erwachsenenbildung stark ein: die öffentlichen Fördermodi heizen nämlich die Marktorientierung und den Wettbewerb unter den Weiterbildungsträgern kräftig an ${ }^{10}$, indem sie belohnen, was gut geht - und das angesichts einer überaus bescheidenen finanziellen Ausstattung des vierten Bildungsbereichs.

In Bayern sieht das etwa so aus, daß der vom Freistaat ausgeworfene Gesamtzuschuß unter den sieben dort anerkannten Trägern öffentlicher Weiterbildung nach der Zahl der in 90 Minuten Veranstaltung Anwesenden verteilt wird. Veranstaltungen, die besonders viele Teilnehmer haben, sind auch besonders zuschußträchtig. Die thematisierten Inhalte spielen in diesem Fördersystem keine Rolle, sofern die 
Thematik innerhalb der vom Gesetz gezogenen inhaltlichen Grenzen angesiedelt ist, und die sind sehr weit gezogen.

Anderswo gilt die durchgeführte Unterrichtsstunde mit Mindestteilnehmerzahl als Bemessungseinheit. Hier mag der Zwang zur Nachfrageorientierung nicht ganz so groß sein wie etwa beim Kriterium der Teilnehmerdoppelstunde in Bayern. Aber auch bei diesem Modus der Zuschußvergabe kommt besonders gut in der Förderung weg, wer besonders viele Doppelstunden „verkauft" hat. Kommt hinzu, daß sich ein Träger im Blick auf etwaige politische Neuordnungen der Weiterbildungslandschaft ein umso größeres Gewicht verschafft, je mehr „Leistung“ gemessen in Veranstaltung, Doppelstunden und Teilnehmern er vorzuweisen hat - Zwänge, denen sich diejenigen, die die Arbeit an der Basis fördern wollen und sollen, auf die Dauer nur schwer entziehen können.

Daß der Gesetzgeber die zum Ausbau des Weiterbildungsbereichs gewährte Förderung so eng mit qualitätsbeeinträchtigenden Wettbewerbszwängen koppelt und damit innovativem Engagement stark die Hände bindet, hat sich die katholische Erwachsenenbildung freilich ein gutes Stück selbst zuzuschreiben. Zusammen mit den anderen freien Trägern - häufig sogar als deren Wortführer - beharrt sie seit Jahren in der Diskussion um die Zukunft des Weiterbildungsbereichs unverrückbar auf dem Stand punkt, daß der Interessen- und Wertepluralität der demokratischen Gesellschaft eine pluralistische Verfassung der Weiterbildung zu entsprechen habe, die in der Vielfalt uneingeschränkt programmautonomer, gleichberechtigter und bei gleicher Leistung gleichgeförderter Träger ihren Ausdruck finden müsse.

Eine solche Position rückt zwangsläufig das Unterscheidende, das Trennende in den Vordergrund, erzeugt Konkurrenz, wo häufig gar keine nötig wäre. Denn sie fußt auf der empirisch nicht haltbaren Prämisse, daß entlang den Grenzen der vorhandenen Weiterbildungslager die faktische Pluralität der gesellschaftlichen Interessen und Anschauungen, der Menschen- und Weltbilder verlaufe, oder daß die jeweilige Trägergruppe ein monolithischer Block sei und sich weltanschaulich total und scharf von der Bezugsgruppe anderer Träger abgrenzen lasse. Sie ist auch schwerlich in Deckung zu bringen mit jener konziliaren Auffassung, die außerhalb der Kirche viel vom Geist des Evangeliums am Werke sieht, ohne daß dieses Handeln ausdrücklich vom Geist Christi her motiviert wäre ${ }^{11}$.

Solange die mit dem undifferenzierten Pluralismusargument erzwungene liberalmarktwirtschaftliche Ordnung des vierten Bildungsbereichs mit dem freien Spiel der Kräfte bei stark unterentwickelter Weiterbildungsmotivation in der Bevölkerung aufrechterhalten wird, muß man die Chance für eine stärker am objektiven gesellschaftlichen Bedarf statt an der Nachfrage orientierte Programmplanung als sehr gering einschätzen. In einem so strukturierten Weiterbildungssystem zahlen sich Investitionen für eine Verbreiterung und Intensivierung sachgerechter Medienerziehung im buchstäblichen Sinn nicht aus. Ein massiv verstärktes medienpädagogisches Engagement geriete eher zum Verlustgeschäft ${ }^{12}$. Das Wachstum, das gebraucht wird, um wenigstens den bisherigen Besitzstand zu wahren oder gar noch einige Marktanteile zuzulegen, läßt sich auf anderen derzeit publikumsattraktiveren Feldern wesentlich leichter erarbeiten. Insofern werden von den mittleren und höheren Ebenen katholischer Erwachsenenbildung kaum die massierten Impulse ausgehen, die zu entscheidenden Veränderungen im Programmverhalten an der Basis führen könnten. 


\section{Folgerungen und Forderungen}

Sicher kommt zu den genannten Mißständen, Schwächen und Rahmenbedingungen auch der Mangel an für die Realisierung sachgerechter Medienerziehung qualifizierten Dozenten hinzu, und als seine Folge auch ein entsprechend dünnes Angebot an medienpädagogischen Veranstaltungsvorschlägen. Die Vergrößerung des Referentenreservoirs, an der das Referat Kommunikationspädagogik bei der Zentralstelle Medien der Deutschen Bischofskonferenz seit Jahren mit einem relativ intensiven Schulungsprogramm arbeitet, wird wohl mit der Zeit das Potential von medienpädagogischen Anregungen vergrößern. Und da und dort dürfte es dann auch einen entsprechenden Niederschlag im Programm finden.

Den Durchbruch zu einer konzeptionell durchdachten, systematischen, kontinuierlichen, breit angelegten Medienerziehung gerade in jenen Bevölkerungsschichten, die sich gegenüber dem Angebot der Bildungshäuser abstinent zeigen, wird man realistischerweise vom langsam wachsenden Angebot an kommunikationspädagogisch geschulten Referenten nicht erwarten dürfen. Eine entscheidende Verkürzung der Kluft zwischen Anspruch und Wirklichkeit der Medienerziehung in der katholischen Erwachsenenbildung wird sich angesichts des komplexen Bedingungsgeflechts der Programmpraxis nicht so sehr über isoliert auf die Verstärkung des medienpädagogischen Engagements zielende Maßnahmen realisieren lassen.

Die Probleme und Schwächen der Medienerziehung in der katholischen Erwachsenenbildung sind die Probleme und Schwächen der Erwachsenenbildung in katholischer Trägerschaft überhaupt und ihres Ordnungskontexts. Medienerziehung wird deshalb nur in dem Maß auch faktisch den Platz finden, den sie in Absichtserklärungen innehat, in dem katholische Erwachsenenbildung die strukturellen und theoretischen Schwächen und Fragwürdigkeiten abstreift und die politischen Rahmenbedingungen verändern hilft, die sie daran hindern, so zu sein, wie sie sein will oder im Dienst der Menschen sein soll.

Konkret heißt das u.a.:

- Mehr und bessere Medienerziehung im Veranstaltungsangebot der katholischen Erwachsenenbildung setzt zunächst einmal voraus, innerkirchlich wie außerhalb entschieden gegen die vorherrschende Verengung des Leistungs- und Erfolgsbegriffs von Bildungsmaßnahmen auf rein quantitative Merkmale anzukämpfen.

- Insbesondere müßte ein umfassendes, theoretisch solides Fortbildungsprogramm für die mittleren und höheren Führungskräfte im katholischen Weiterbildungslager initiiert werden, damit Medienerziehung stärker als bisher als eine ganz zentrale Aufgabe der Lebens- und Orientierungshilfe in deren Bewußtsein gehoben und von dort den Programmplanern vor Ort weitervermittelt wird.

- Mehr und bessere Medienerziehung setzt desweiteren eine gezielte Personalpolitik voraus. Im Hauptamtlichenstab auf Diözesan-Ebene sollte jeweils ein medienund erziehungswissenschaftlich Ausgebildeter die Funktion der Interessenvertretung der Medienerziehung nach innen, in die eigene Organisation hinein, wahrnehmen.

- Jene vom Kulturbeirat des Zentralkommitees der deutschen Katholiken geforderte Programmvereinheitlichung durch die Vorgabe curricularer Mindest- 
standards ${ }^{13}$ könnte der Medienerziehung in der flächendeckenden Erwachsenenbildung mehr Geltung verschaffen. Auf jeden Fall erscheint es unumgänglich, die fragwürdige totale Programmautonomie der überforderten örtlichen Planungsinstanzen etwas einzuschränken, ohne eine durchgängige Zentralisierung der Bildungsarbeit einzuführen.

- Mit der Errichtung einer pädagogischen Arbeitsstelle der katholischen Erwachsenenbildung auf Bundes- und entsprechenden eng zusammenarbeitenden Einrichtungen auf Landesebene wäre ein Kanal zu schaffen, über den das Referat Kommunikationspädagogik bei der Medienzentrale der Deutschen Bischofskonferenz das Anliegen Medienerziehung in die Praxis der Erwachsenenbildung erfolgversprechender einspeisen könnte als in der gegenwärtigen Unverbundenheit der verschiedenen Handlungsbereiche und Ebenen.

- Eine ganz entscheidende Voraussetzung für eine Verbesserung der Situation der Medienpädagogik könnte eine Neuordnung des staatlichen und kommunalen, aber auch des binnenorganisatorischen Fördersystems schaffen. Über eine investive Förderung schwer vermittelbarer Bildungsvorhaben, über Sonderzuschüsse usw. ließe sich die Nachfrageorientierung in der Führungsschicht aufbrechen und der Mut zum gewiß schwierigen und aufwendigen Ausbau der so notwendigen Medienpädagogik wecken.

\section{Anmerkungen}

1 Siehe dazu den vom Autor unternommenen Versuch einer Grobanalyse des medienpädagogigschen Angebots im Gesamt der Aktivitäten katholischer Erwachsenenbildung in 1:1981, S. 50-60 dieser Zeitschrift.

2 Zur Orientierung über die in der Sozialpsychologie und Kommunikationsforschung gewonnenen bewährten Einsichten in die Stabilität und Veränderbarkeit der erwachsenen Persönlichkeit: Handbuch der Psychologie, Band 7 Sozialpsychologie, Halbband 2, Göttingen 1972, insbesondere Teil lV.

${ }^{3}$ Dazu: Katholische Bundesarbeitsgemeinschaft für Erwachsenenbildung (Hrsg.): Statistische Berichte 1980, Bonn 1980; Georg Betz: Hoher Aufwand, mäßiger Ertrag. Zur Situation katholischer Erwachsenenbildung, in: „Herder Korrespondenz“, Freiburg, 2:1980, S. 89 f.

${ }^{4}$ Der Lehrstuhl Erwachsenenbildung der Katholischen Universität Eichstätt hat es zu einem Schwerpunkt seines Arbeitsprogramms gemacht, die Situation der katholischen Erwachsenenbildung vor Ort zu erhellen. Bisher liegen dazu zwei Arbeiten vor: Karl Heinz Schoppelrey: Selbstverständnis, Qualifikation und Motivation ehrenamtlicher Mitarbeiter, Unveröffentl. Diplomarbeit, Phil.-Päd. Fakultät der katholischen Universität Eichstätt, 1979; Maria Czech: Strukturen, Verlaufsformen und Einflußfaktoren der Programmplanung vor Ort. Am Beispiel des Katholischen Bildungswerks Stadt Augsburg e.V., Unveröffentl. Diplomarbeit, Phil.- Päd. Fakultät der Katholischen Universität Eichstätt, 1980.

5 Zur Weiterbildungsbereitschaft und -abstinenz vor allem: Wolfgang Schulenberg, HeinzDieter Loeber, Uta Loeber-Pautsch, Susanne Pühler: Soziale Faktoren der Bildungsbereitschaft Erwachsener, Stuttgart 1978; Willi Strzelewicz, Hans-Dietrich Raapke, Wolfgang Schulenberg: Bildung und gesellschaftliches Bewußtsein, Stuttgart 1966.

6 Vgl. Bundesarbeitsgemeinschaft für katholische Erwachsenenbildung/Institut für Erwachsenenbildung: Empfehlungen zur Struktur der örtlichen katholischen Erwachsenenbildung, 1969, Punkt 4. 
7 Franz Pöggeler, einer der langjährigen wissenschaftlichen Begleiter der katholischen Bundesarbeitsgemeinschaft für Erwachsenenbildung kommt zu einem ähnlichen Urteil über ihre jüngere Entwicklung. Er spricht etwas resigniert davon, daß sie ,in mancherlei Hinsicht die Orientierung an der Theorie - der erziehungswissenschaftlichen wie der theologischen - verloren hat und sich auf statistisch meßbaren Aktivismus verlegt hat". Franz Pöggeler: Christliche Erwachsenenbildung in einem pluralistischen Bildungssystem, in: Franz Henrich (Hrsg.): Erwachsenenbildung in der pluralen Gesellschaft. Düsseldorf 1978, S. 135.

${ }^{8}$ Bereits 1973 hat der Kulturbeirat des Zentralkomitees der deutschen Katholiken die ungenügende Verzahnung der Handlungsbereiche als gravierende Schwäche katholischer Erwachsenenbildung moniert. Vgl. Kulturbeirat des Zentralkomitees der deutschen Katholiken: Gutachten zur Weiterbildung in katholischer Trägerschaft und zu einem kooperativen System der Weiterbildung, Bonn 1973, Punkt C 1.

9 Vgl. Peter Kirfel: Aufgaben einer katholischen Bundesstelle für Weiterbildung, in „Erwachsenenbildung“, Osnabrück, 3:1975, S. 134ff.

$10 \mathrm{Zu}$ den Implikationen der Weiterbildungsfinanzierung: Jürgen Kunze: Weiterbildungskonzeptionen und Finanzierungsinstrumente - eine Modellstudie, in: Deutscher Bildungsrat. Gutachten und Studien der Bildungskommission: Umrisse und Perspektiven der Weiterbildung. Stuttgart 1975, S. 146ff.

11 Eine ausführlichere Kritik des Pluralismus-Arguments enthält: Georg Betz: Freier Wettbewerb oder flächendeckendes Angebot? Zum Pluralismus-Konzept in der Erwachsenenbildung, in: „Herder Korrespondenz“, Freiburg, 10:1980, S. $517 \mathrm{ff}$.

12 In Rechnung zu stellen bleibt dabei auch die Anhängigkeit von den Veranstaltungsgebühren der Teilnehmer, die angesichts der unzureichenden öffentlichen Förderung besonders durchschlägt und die Zurückhaltung gegenüber Themen und Veranstaltungen, die „erfahrungsgemäß" nicht so zugkräftig sind, noch erheblich verstärkt. Immerhin finanziert sich katholische Erwachsenenbildung zum Teil bis zu einem Drittel aus Teilnehmergebühren.

13 Vgl. Kulturbeirat des Zentralkomitees der deutschen Katholiken, a.a.O.

\section{SUMMARY}

Except in specialised institutions, commitment to media instruction within the framework of Catholic Adult Education leaves much to be desired, both in quality and quantity. This situation arises partly from the lack of qualified lecturers. Weak and indifferent training schemes seem to stem more generally from weaknesses in structure and theory of the whole education system: decision on the programme is left to cooperators who are not sufficiently competent in this area. Then there is the pressure of achieving success and visible results, and an insufficient theoretical competence on the part of decision makers. There is also a lack of coordination and interlink between the different disciplines as well as the need to ensure a sufficient number of participants in order to obtain appropriate subsidies. This situation can be remedied within an overall theoretical foundation and a more streamlined structure in the whole area of Catholic Adult Education, and a more up-to-date orientation for continued formation, and the situation of media education. 


\section{RÉSUMÉ}

L'engagement pédagogique des media, dans le cadre de la formation pour adultes, d'obédience catholique, en dehors des maisons de formation, laisse, en quantité et en qualité, beaucoup à désirer. Cela ne s'explique pas seulement par le manque de rapporteurs capables. Les faiblesses et objets de contestations de l'offre pédagogique des media se situe surtout dans la formation catholique pour adultes, dans des faiblesses particulièrement caractéristiques de la structure et de la théorie, et dans des mises en question de l'ordre de l'ensemble du domaine de la formation continue: dans l'attribution des décisions concernant le programme destiné aux collaborateurs objectivement surchargés, dans la notion de rendement et de reussite fortement définie quant à la quantité, dans la compétence théorique insuffisante des cadres, dans l'engrenage et la coordination insuffisants des domaines d'action, dans les conditions de subvention renforçant encore l'orientation de la demande. La situation de l'éducation des media ne peut s'améliorer que dans le cadre d'un fondement théorique vaste et d'une structuration plus ferme de l'ensemble de la pratique de la formation catholique pour adultes, de même qu'une réorganisation plus objective de l'ensemble du paysage de la formation continue.

\section{RESUMEN}

El comprometimiento de los medios pedagógicos en el campo de la formación católica de adultos, al margen de los centros de formación, deja mucho que desear en cantidad y calidad. Esta deficiencia no es solo achacable a la falta de personal cualificado. El punto débil de la oferta pedagógica en la formación católica de adultos se encuentra más bien, estructural y teóricamente, en la debilidad y falta de crédito de todo el sistema de formación de adultos: en la delegación de las decisiones sobre el programa a colaboradores objetivamente desbordados en sus capacidades, en conceptos de rendimiento dominados por criterios cuantitativos, en la insuficiente competencia teórica de los directivos, en el deficiente ensamblaje y la magra coordinación de las materias, en la mecánica de las subvenciones, que deberían orientarse más que hasta ahora por la demanda. Solo en el marco de una reestructuración radical y una reorientación teórica de toda la práctica de la formación católica de adultos, mediante un reordenamiento de la panorámica de la formación, podrá mejorarse la situación de los medios educativos. 